\section{Topische Therapie bei Psoriasis: Rezepte enthalten zu wenig oder falsche Angaben}

Patienten mit leichter bis mittelstarker Psoriasis werden meist topisch behandelt. Doch die Wirkung der Substanzen ist oft ungenügend. Das liegt nicht unbedingt an der schlechten Compliance der Patienten oder einem Therapieversagen. In einer französischen Studie zeigte sich: Zwei Drittel aller Rezepte enthielten nicht die erforderlichen Informationen, die ein Psoriasis-Patient benötigt, um das verschriebene Präparat richtig anwenden zu können.

$D$ ie Kommunikation zwischen Arzt und Patient lässt zu wünschen übrig. Einer früheren Studie zufolge verstehen $80 \%$ der Patienten die mündlichen Ausführungen ihres Arztes falsch, wenn er ihnen ein neues Medikament verschreibt. In einer aktuellen systematischen Analyse wurde nun die Qualität von 767 Verschreibungen topischer Substanzen durch Dermatologen und Allgemeinärzte unter die Lupe genommen. Bewertet wurden folgende Parameter: die Art der Verschreibung (per Computer oder handgeschrieben), die Indikation, die Häufigkeit der Anwendung, die Behandlungsdauer, die Beschreibung der zu behandelnden Areale sowie Angaben zur Dosierung. Als Verschreibungen von hoher Qualität wurden diejenigen bewertet, die mindestens vier der genannten fünf Qualitätsparameter erfüllten.
53\% der Rezepte waren von Dermatologen ausgestellt, $47 \%$ von Allgemeinärzten. Bei knapp der Hälfte handelte es sich um den Beginn einer Behandlung. 63\% der Rezepte waren handgeschrieben, 37\% elektronisch erstellt. Die Anforderungen an eine qualitativ hochwertige Verschreibung erfüllten nur 35,7\% der Rezepte. Ein Viertel der Verschreibungen berücksichtigten sogar nur maximal zwei der Qualitätskriterien.

Die Qualität wurde insgesamt von zwei Faktoren signifikant beeinflusst: Art der Rezeptausstellung - elektronisch ausgestellte Rezepte waren besser (OR: 3,04; $\mathrm{p}<0,0001)$ und Fachrichtung des verschreibenden Arztes - Dermatologen verschrieben bessere Rezepte als Allgemeinärzte (OR: 1,61, 95\%; p < 0,0001).

Bei 26\% der Rezepte wurde die topische Verwendung des Präparats nicht erwähnt, in $18 \%$ der Fälle waren keine Angaben darüber zu finden, wie oft der Patient das Präparat auftragen soll, bei $77 \%$ wurde das zu behandelnde Areal nicht definiert und bei 55\% fehlte die Angabe zur Packungsgröße.

Fazit: Die Qualität der Verschreibung topischer Präparate könnte verbessert werden, indem die Rezepte per Computer ausgestellt werden. Zudem sollten Hilfen für den Arzt entwickelt werden, um die benötigte Dosis eines topischen Wirkstoffs der betroffenen Körperfläche besser anzupassen. Vor allem aber brauchen die Patienten eine klare schriftliche Anleitung, damit sie die Substanzen richtig anwenden. Idealerweise sollten auf dem Rezept für ein topisches Präparat Menge und Häufigkeit der Applikation angegeben werden. Außerdem ist die zu behandelnden Areale genau zu bereichnen.

Dr. Christine Starostzik

Pouplard C et al. Are we giving patients enough information on how to use topical treatments? Analysis of 767 prescriptions in psoriasis. Brit J Dermatol 2011; Jun 28 [Epub ahead of print]

\title{
Interessanter Nebenbefund: Kalkhoden
}

Ein 32-jähriger Mann suchte die Nothilfe wegen Dyspnoe und Hämoptysen auf. Bei der körperlichen Untersuchung fielen weißlich-gelbe Knötchen in der Skrotalhaut auf.

$\mathrm{D}$ ie indolenten derben Knötchen (Abb. A) erlaubten die klinische Blickdiagnose einer Calcinosis scrotalis.

Im Kontrastmittel-CT des Thorax und des Abdomens fanden sich zahlreiche kleine Lungenemboli, woraufhin die Ärzte eine Antikoagulation einleiteten. Im Becken-CT stellten sich die intrakutan liegenden verkalkten Knoten der Skrotalhaut in einer Größe von 3-8 mm dar (Abb. B, Pfeil).

Kommentar: Bei der Calcinosis scrotalis handelt es sich um eine gutartige Veränderung ungeklärter Ätiopathogenese. Störungen des Kalzium- oder Phosphat- stoffwechsels liegen nicht vor. Die intradermal gelegenen Knoten nehmen meist im Laufe von Jahren an Größe zu und können exulzerieren, sodass sich weißliches, kreideartiges Material entleert. Die meisten Patienten wollen die Knoten aus ästhetischen Gründen entfernt haben.

Prof. Dr. Hermann Füeß।

Grenader T, Shavit L. Scrotal calcinosis. New

Engl J Med 2011; 365: 647
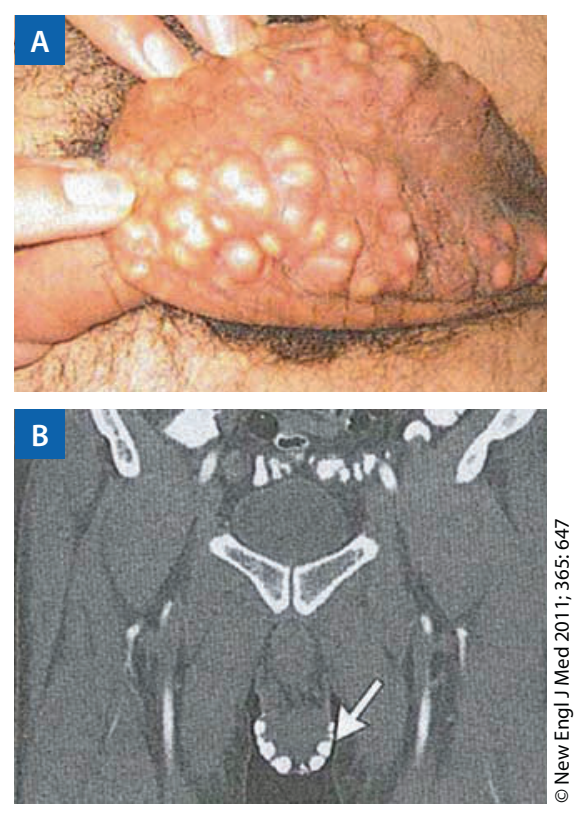

Calcinosis scrotalis: klinisches Bild (A) und $\mathrm{CT}$ (B). 